\title{
NOVEL CONCEPTS FOR HIGH-EFFICIENCY LIGHTWEIGHT SPACE SOLAR CELLS
}

\author{
F. Cappelluti( ${ }^{(1)}$, G. Ghione ${ }^{(1)}$, M. Gioannini( ${ }^{(1)}$, G. Bauhuis ${ }^{(2)}$, P. Mulder $^{(2)}$, J. Schermer $^{(2)}$, M. Cimino $^{(3)}$, G.

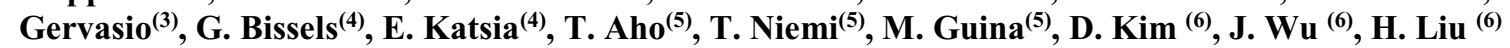 \\ (I) Department of Electronics and Telecommunications, Politecnico di Torino, \\ Cso Duca degli Abruzzi 24, 10129 Torino, Italy, \\ Email: federica.cappelluti@polito.it \\ (2) Institute for Molecules and Materials, Radboud University, Heyendaalseweg 135, 6525 AJ Nijmegen, The \\ Netherlands,Email:g.bauhuis@science.ru.nl,p.mulder@science.ru.nl,j.schermer@science.ru.nl \\ (3) Thales Alenia Space, Strada Antica di Collegno 253, 10146 Torino (Italy), \\ Email: Giuseppe.Gervasio@thalesaleniaspace.com \\ (4) tf2 devices B.V., Heyendaalseweg 135, 6525 AJ Nijmegen, The Netherlands, \\ Email:gunther.bissels@tf2devices.com,eleni.katsia@tf2devices.com \\ (5) Optoelectronics Research Centre,Tampere University of Technology, Korkeakoulunkatu3,33720,Tampere, \\ P.O.Box692, 33101Tampere, Finland, \\ Email: mircea.guina@tut.fi \\ ${ }^{(6)}$ Department of Electronic and Electrical Engineering, University College London, Torrington Place, \\ London WC1E 7JE, United Kingdom, \\ Email: huiyun.liu@ucl.ac.uk
}

\begin{abstract}
One of the key issues in the design and development of a satellite Photovoltaic Assembly (PVA) is the trade-off to be made between the available volume located to the PVA, its mass and the total amount of power that the solar panels have to guarantee to the spacecraft. The development of high-efficiency, flexible, lightweight solar cells is therefore instrumental to the design of future satellites providing enhanced missions and services. Based on the consolidated development of GaAs-based single junction and lattice matched triplejunction solar cells, several research efforts are being pursued worldwide to further increase the efficiency and reduce mass. Promising approaches include thin-film technologies such as Inverted Metamorphic and Epitaxial Lift-Off (ELO), and the use of nanostructures or highly mismatched alloys grown by MBE. We propose here an alternative path towards the development of lightweight GaAs-based solar cells with the potential to exceed the Shockley-Queisser (SQ) limit of single junction cells. Our approach is based on the synergistic combination of thin-film design, quantum dots (QDs) absorption, and photonic nanostructures. Challenges and opportunities offered by the use of QDs are discussed. A cost-effective and scalable fabrication process including ELO technology and nanoimprint lithography is outlined. Finally, a proof-of-concept design, based on rigorous electromagnetic and physicsbased simulations, is presented. Efficiency higher than $30 \%$ and weight reduction close to $90 \%$ - owing to the substrate removal - makes the proposed device to rank record power-to-weight ratio, with the potential to become a cost-effective, attractive option for next generation space solar cells.
\end{abstract}

\section{INTRODUCTION}

Today's industry standard for space solar arrays is provided by triple-junction III-V solar cells on a GaAs or Ge substrate having efficiency of $30 \%$. In these cells the typical substrate thickness is about 150-200 $\mu \mathrm{m}$ (notice that the useful active device thickness for photovoltaic conversion is less than $5 \mu \mathrm{m}$ ), resulting in an average weight of the bare solar cell close to 90 $\mathrm{mg} / \mathrm{cm}^{2}$ and a power-to weight ratio of about $400 \mathrm{~W} / \mathrm{kg}$. Besides their large weight, such cells are not flexible. Indeed, the development of flexible high efficiency solar cells will enable the design of new solar arrays architectures such as rollable solar arrays or inflatable ones with the possibility to be used in satellites (commercial and scientific) or moon bases. At the satellite level, increased power-to-weight ratio and flexibility may allow for knock-on advantages in terms of launch cost savings or payload enhancement or higher performances, depending on the demands of the specific application. Thus, there is a strong effort worldwide to develop new III-V cell technologies aiming at higher efficiency and lower mass. In these approaches, the wafers only serve as a production template and are removed to obtain genuine thin-film solar cells.

Besides remarkable mass and cost reduction (if wafer reuse is possible) the thin-film approach is theoretically superior since it allows for a substantial increase of efficiency in radiative-limited cells, like the GaAs-based one, through photon recycling. Thin-film configurations with back-side mirrors have been the key to the most recent efficiency record of $28.8 \%$ [1]. By further optimization of the back reflector, an efficiency higher than $30 \%$ is within reach. Nano-engineering of the semiconductor material might yield even higher 
efficiency with a less complex technology than multijunction cells. Quantum dot cells have been extensively investigated, motivated by the idea of extending the photon harvesting at longer wavelengths and improve the short circuit current taking advantage of subbandgap optical transitions enabled by QDs. However, one of the main shortcomings of this approach is the small QD optical cross-section, that has prevented up to now the demonstration of a significant efficiency increase with respect to single-junction cells. In this respect, the thin-film geometry is a prerequisite to the implementation of light trapping techniques, that can allow for a large enhancement of the QD short circuit current. In particular, nanostructuring the bottom or top wide-gap window layers, combined with suitable reflectors and Anti-Reflection Coating (ARC) layers, may provide efficient light trapping within the absorbing layers. Nanostructuring of the thin-film cell may be realized through a cost-effective technology such as Nanoimprint Lithography (NIL) that allows to pattern even subwavelength periodic gratings over large areas.

\section{TECHNOLOGY}

\subsection{THIN-FILM FLEXIBLE CELLS BY EPITAXIAL LIFT- OFF}

For the current generation of $\mathrm{InGaP} / \mathrm{GaAs} / \mathrm{Ge}$ triple junction cells, the Ge wafer on which they are produced is an integral part of the cell structure. However, for the next generation solar cells the $\mathrm{Ge}$ (or GaAs) wafers might only serve as a production template that can be removed to obtain genuine thin-film solar cells. The enabling technology for this approach will be the Epitaxial Lift-Off (ELO). ELO allows removal of the III-V photo-sensitive layers in such a way that the wafers can be reused.

For this purpose, a typically $10 \mathrm{~nm}$ thick $\mathrm{Al}_{\mathrm{x}} \mathrm{Ga}_{1-\mathrm{x}} \mathrm{As}(\mathrm{x}$ $>0.6$ ) release layer is deposited before the actual III-V cell structure. This sacrificial layer allows for the separation of the device structure from its native semiconductor wafer by selective wet etching using an aqueous HF solution [2]. The thin, single crystal cell structures obtained by the ELO technology can be mounted on low-cost foreign carriers, like metal or plastic foils, to achieve stability during further processing. The fact that these carriers can be selected on the basis of their material properties rather than of crystal growth demands, opens the way for the development of innovative device structures. Owing to the large selectivity $\left(>10^{6}\right)$ of the HF solution for etching of $\mathrm{Al}(\mathrm{Ga}) \mathrm{As}$ over $\mathrm{GaAs}$ and $\mathrm{Ge}$, the expensive semi-conductor wafer is not affected, and can be reused as a production platform for subsequent thin-film cells [3].

ELO involves lateral etching of the release layer. Nevertheless, increasing the size of the structures subjected to the process does not impose fundamental problems, as long as the released part of the thin-film structure is bent away during the process, leaving the etch front easily accessible for the etch solution [2]. In this way, the process was up-scaled from (typically) 1 $\mathrm{cm}^{2}$ samples to complete 2" diameter and further to 4" diameter wafers (see Fig. 1). It was furthermore demonstrated that the technology can be applied to III-V cells from any supplier of epi-structures if the thin-film structure on the wafer is properly prepared to enable the ELO process step.

Besides reusing the semiconductor wafers, the ELO technology also enables the production of unique thinfilm device geometries that allow for a substantial increase in cell efficiency by photon recycling. Since GaAs solar cells are limited by radiative recombination, the application of a back-contact/mirror thin-film cell geometry resulted in thin-film GaAs cells with a world record efficiency of $26.1 \%$ in 2009 [4]. Subsequently, theoretical studies indicated that additional improvements in the back-side mirror reflectivity beyond $95 \%$ will increase the cell efficiency superlinearly [5], resulting in the current $28.8 \%$ efficiency record reported by Alta Devices [1]. Further improvements in this respect have the potential to yield single-junction thin-film GaAs cells with an efficiency exceeding $30 \%$.

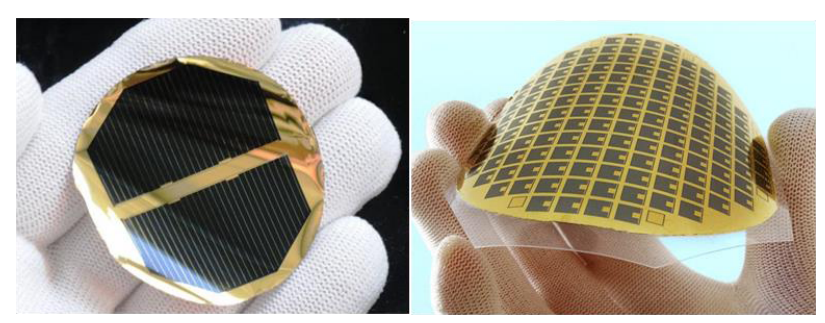

Figure 1. Two high-efficiency, thin-film III-V cells on a 2" diameter metal foil acting as mirror and back contact produced at Radboud University (left) and a large number of small thin-film III-V cells for concentrator system applications produced on a 4" diameter foil at tf2-devices (right).

\subsection{QUANTUM DOT MATERIAL}

For the implementation of high-efficiency QDSCs, it is essential to achieve a substantial improvement in the optical absorption of QD arrays by increasing the number of QDs. To this end, Sugaya et al. have reported ultra-high stacked InGaAs/GaAs QDSCs [6]. A 400stack QD structure was grown without using a strain balancing technique, which led to the total QD density of $2 \times 10^{13} \mathrm{~cm}^{-2}$. Also, for high in-plane QD density, we have previously demonstrated Sb-mediated InAs QD growth [7]. In-plane QD density of $1 \times 10^{11} \mathrm{~cm}^{-2}$ was achieved by depositing Sb before the QD growth, which led to an increase in the photon absorption, and hence an improvement in the short-circuit current.

In this work, we have grown a high-stack (100-stack) 
InAs/GaAs QD structure with high in-plane QD density, as shown in Fig. 2. High in-plane QD density $\left(8 \times 10^{10}\right.$ $\mathrm{cm}^{-2}$ ) is achieved by applying the Sb-mediated QD growth technique, as previously demonstrated [7]. The AFM images in Fig 3 show that the QD structure has a good surface morphology and high in-plane QD density even after 100 stacks of QD layers.

\begin{tabular}{|c|c|}
\hline $16.5 \mathrm{~nm} \mathrm{GaAs}$ & $\llcorner 9 \mathrm{ML} \mathrm{Sb}$ \\
\hline $\begin{array}{r}3.5 \mathrm{~nm} \text { GaAs } \\
\boldsymbol{\Delta} \boldsymbol{\Delta} \boldsymbol{\Delta} \boldsymbol{\Delta} \boldsymbol{\Delta} \boldsymbol{\Delta} \boldsymbol{\Delta} \\
\end{array}$ & $\begin{array}{l}\text { - } 2.275 \mathrm{ML} \ln A s \mathrm{QDs} \\
\boldsymbol{\Delta} \boldsymbol{\Delta} \boldsymbol{\Delta} \boldsymbol{\Delta} \boldsymbol{\Delta} \boldsymbol{\Delta} \boldsymbol{\Delta} \boldsymbol{\Delta}\end{array}$ \\
\hline $50 \mathrm{~nm} \mathrm{GaAs}$ & ᄂ $9 \mathrm{ML} \mathrm{Sb}$ \\
\hline \multicolumn{2}{|c|}{$200 \mathrm{~nm}$ GaAs buffer } \\
\hline $\mathrm{n}^{+} \mathrm{GaAs}(001)$ & trate \\
\hline
\end{tabular}

Figure 2. Structure of the high-stack (100-stack) InAs/GaAs QD structure with high in-plane QD density using $S b$-mediated growth technique.
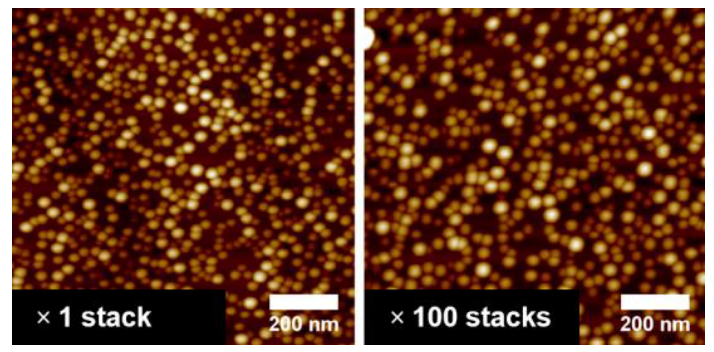

Figure 3. AFM images of InAs QDs (Sb-mediated growth) for 1-stack (left) and 100-stack (right) InAs/GaAs QD structures.

\subsection{NANOSTRUCTURES FOR PHOTONIC MANAGEMENT}

Nanophotonic gratings will be realized implementing a process already applied for demonstrating moth-eye broad-band antireflection coatings (ARC) [8].

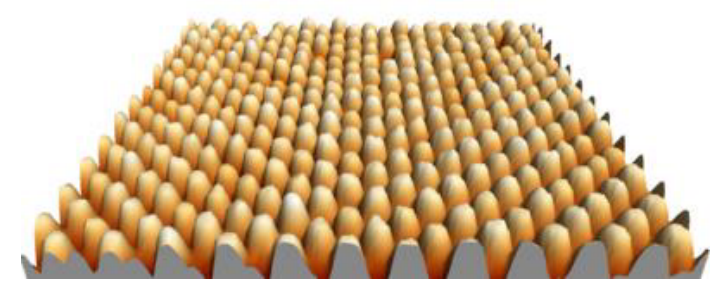

Figure 4. Nanostructured AlInP acting as a broad-band ARC for a GaAs-based solar cell.

In particular, we will use a fabrication process based on replication by Nanoimprint Lithography (NIL) to pattern even subwavelength period gratings over large areas. When employing the NIL technique, the main component of the overall process cost, besides labour and capital expenses, is the cost of the wafer-masters, that is around 2000-3000€/master. Since the master can be replicated up to $\sim 100$ times, mass production enabled by NIL can bring the cost of nano-structuring down to few euros per wafer. Using NIL, wafers with size up to 6" can be patterned in single step. Moreover, since NIL can be integrated as a part of the normal process flow to define contact-grids, the NIL process is deemed to be significantly cheaper (also in terms of scalability) than the standard deposition of dielectric films.

When it comes to defining light-trapping by microsstructuring the back contact (which will also act as a reflector) we will pay special attention to the choice of metals forming the contact layer and to the microstructuring strategy, to ensure high reflection without sacrificing the electrical performance of the contact. A preliminary experiment showing the role of the metals forming the back contact reflector is shown in Fig. 5 [9].

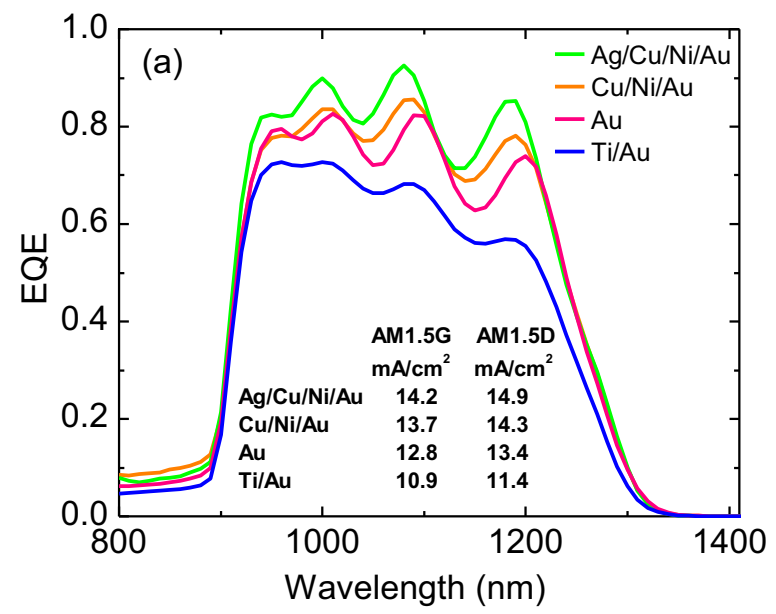

Figure 5 External quantum efficiency for 1-eV solar cell with a back contact reflector formed of different metal combinations. Inset shows the equivalent current generation.

\section{PROOF-OF-CONCEPT DESIGN}

In this section we discuss, as a proof-of-concept, the design of a realistic configuration for a thin-film InAs/GaAs QD cell with light-trapping and photonrecycling, that makes use of the technological approaches described so far. The design is carried out through rigorous electromagnetic simulations, based on Finite-Difference Time-Domain (FDTD) and Rigorous Coupled Wave Analysis (RCWA), and QD-awaretransport simulations [11].

\subsection{ELECTRICAL STRUCTURE AND MODEL PARAMETERS}

To focus the ideas we consider a deep junction design with a thick lightly doped emitter and a thin base layer. 
In high quality materials, the deep junction design has been shown to achieve higher open circuit voltage and is particularly suited for taking advantage of the photon recycling effect [13]. A sketch of the device crosssection and the associated energy band diagram at shortcircuit condition is shown in Fig. 6. A stack of 20 InAs/GaAs QD layers - embedded in a $420 \mathrm{~nm}$ thick intrinsic region - is placed at the bottom of the lightly doped emitter region, next to the junction, in order to maximize carrier collection efficiency at short-circuit.
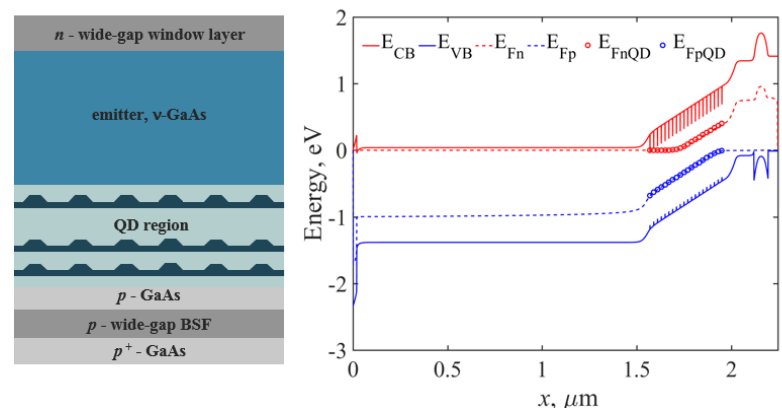

Figure 6. Left: Cross-section of the analysed QD-based solar cell. An $n^{+}$GaAs cap layer (not shown) is used for the realization of the top metal contact. Right: Calculated energy band diagram at short-circuit condition under AM1.5G illumination.

Electrical simulations are carried out by including in a classical drift-diffusion-Poisson formulation the detailed description of charge carrier transitions in the QD states. To this aim, QDs are modelled as a three-level system, including electron/hole ground state (GS), excited state (ES) and the two dimensional wetting-layer state (WL). Carrier exchange among QD states and with bulk states occurs through optical and thermal excitation and relaxation processes. The characteristic scattering times and the optical absorption properties assumed for the QD material are summarised in Table 1 and Fig. 7, respectively. As seen in Fig. 7, QDs sub-bandgap states extend the harvested wavelength range beyond the GaAs band edge. However, due to the low absorption of these states, some light management scheme need to be employed to reach a significant enhancement of the short-circuit current, as discussed in Sec. 3.2.

The model also includes barrier radiative recombination, with radiative coefficient of $2.0 \times 10^{-10}$ $\mathrm{cm}^{3} \mathrm{~s}^{-1}$, and defect induced non-radiative recombination modelled according to Shockley Read Hall (SRH) theory, with characteristic lifetime of $0.5 \mu \mathrm{s}$. In high quality GaAs crystal, SRH lifetimes as high as $1 \mu$ s are achievable [10]. All the presented simulations are done at ambient temperature, $\mathrm{T}=300 \mathrm{~K}$. Photovoltaic characteristics are computed under 1 sun AM1.5G illumination.

Further details on the modelling approach may be found in [11], [12].

\section{Table 1. QD parameters}

\begin{tabular}{l|c}
\hline QD density, $N_{\mathrm{QD}},\left[\mathrm{cm}^{-2}\right]$ & $6 \times 10^{10}$ \\
State degeneracy, $g_{\mathrm{ES}}^{e, h}, g_{\mathrm{GS}}^{e, h}$ & 4,2 \\
WL Density of States, $N_{\mathrm{WL}}^{e, h},\left[\mathrm{~cm}^{-2}\right]$ & $2.4 \times 10^{12}$ \\
$\Delta E_{\mathrm{B}-\mathrm{WL}}^{e}, \Delta E_{\mathrm{WL}-\mathrm{ES}}^{e}, \Delta E_{\mathrm{ES}-\mathrm{GS}}^{e},[\mathrm{meV}]$ & $40,162,70$ \\
$\Delta E_{\mathrm{B}-\mathrm{WL}}^{h}, \Delta E_{\mathrm{WL}-\mathrm{ES}}^{h}, \Delta E_{\mathrm{ES}-\mathrm{GS}}^{h},[\mathrm{meV}]$ & $4,40,16$ \\
$\tau_{\mathrm{CAP}, \mathrm{B} \rightarrow \mathrm{WL}}^{e}, \tau_{\mathrm{CAP}, \mathrm{WL} \rightarrow \mathrm{ES}}^{e}, \tau_{\mathrm{CAP}, \mathrm{ES} \rightarrow \mathrm{GS}}^{e},[\mathrm{ps}]$ & $0.3,1.2,1.2$ \\
$\tau_{\mathrm{CAP}, \mathrm{B} \rightarrow \mathrm{WL}}^{h}, \tau_{\mathrm{CAP}, \mathrm{WL} \rightarrow \mathrm{ES}}^{h}, \tau_{\mathrm{CAP}, \mathrm{ES} \rightarrow \mathrm{GS}}^{h},[\mathrm{ps}]$ & $0.1,0.1,0.1$ \\
$\tau_{r}^{\mathrm{WL}}, \tau_{r}^{\mathrm{ES}}, \tau_{r}^{\mathrm{GS}},[\mathrm{ns}]$ & $1,1,1$ \\
\hline
\end{tabular}

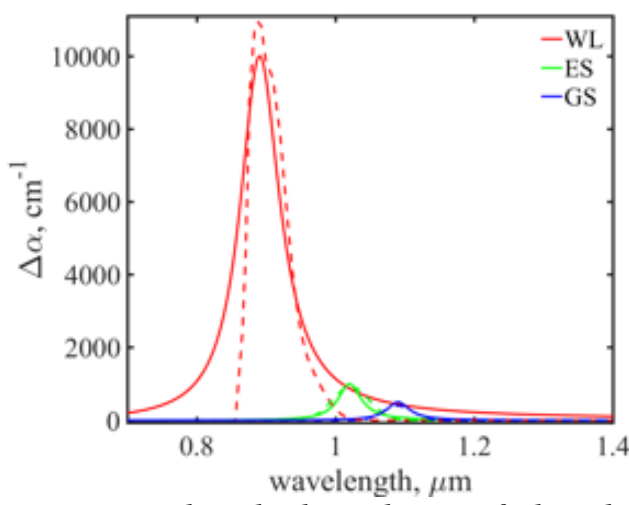

Figure 7. Wavelength dependence of the absorption coefficient of the QD material as estimated by literature data (solid lines) and approximated by Lorentz oscillators for the sake of electromagnetic simulations (dashed lines).

\subsection{Photonic STRUCTURES DESIGN}

For the sake of illustrating the impact of photon management on the QD cell performance, we describe a couple of representative designs: i) a thin-film device combining a nanostructured periodic grating on the top surface with a planar reflector at the rear side; ii) a thinfilm device with planar antireflection coating and a periodic grating on the rear side. The first design aims at reaching very low reflectivity over the whole GaAs and QD wavelength range and doubling photocurrent at sub-bandgap wavelengths. The second design aims at investigating the achievable QD photocurrent enhancement through light-trapping schemes.

A sketch of the first structure is reported in Fig.8 (a): the top-surface periodic grating acts as broadband antireflection coating (ARC), with very low reflectivity in the 400-1200 $\mathrm{nm}$ range. The top-surface grating may also provide some light-trapping enhancement in the wavelength range of about $800-1100 \mathrm{~nm}$ by exciting higher order diffraction modes. The presented results refer to the ARC-optimized geometry realized in [8], with nanocone size features of 80/300/440 nm (top diameter /base diameter /height). With such dimensions, reflectivity well below $5 \%$ is achieved in a broadband 
range covering the GaAs band edge and the QD interband transitions [8].

We estimated the absorption enhancement (Fig. 3 and Table 2) in the GaAs/QD active region through FDTD simulations. Fig. 8 compares the absorbed photon for various device configurations: i) a conventional, waferbased single-pass cell with planar ARC; ii) a waferbased cell with nanostructured ARC (the nanocone); iii) a thin-film cell with nanostructured ARC and ideal reflector (modelled as perfect electrical conductor).
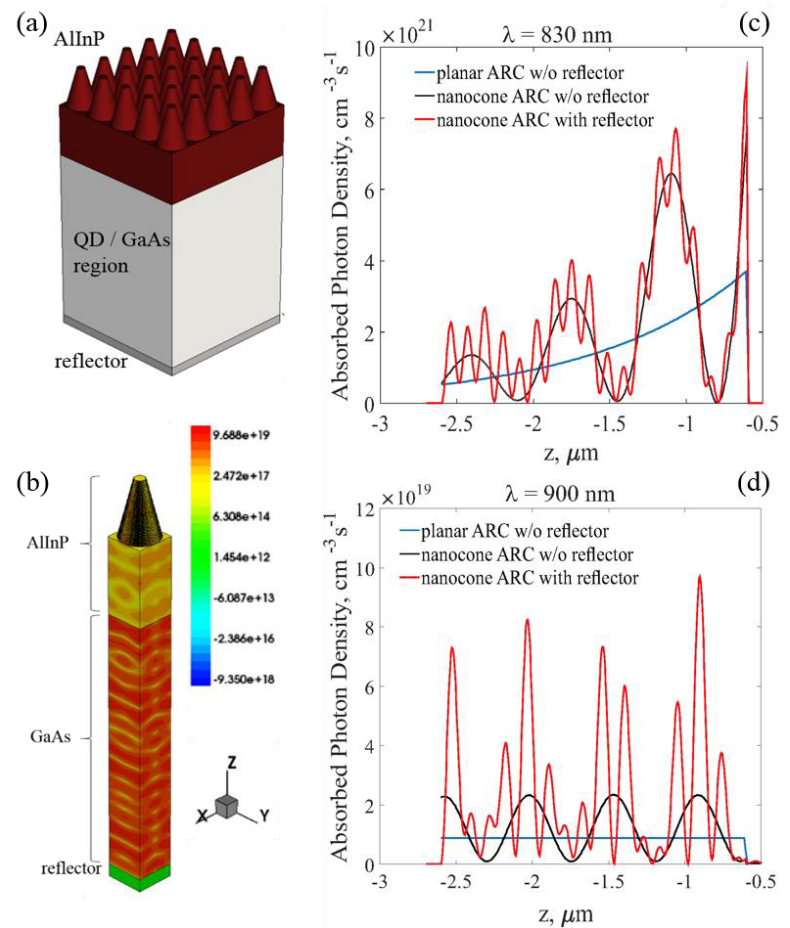

Figure 8. (a) Conceptual sketch of solar cell exploiting nanostructured ARC and reflector. (b) $3 D$ distribution of the absorbed photon density $\left(\mathrm{cm}^{-3}\right)$ at $\lambda=900 \mathrm{~nm}$ for the device with nanocone ARC and planar reflector. (c), (d) Cutline across the z-axis of the absorbed photon density at $\lambda=830 \mathrm{~nm}$ and $\lambda=900 \mathrm{~nm}$, respectively, for the various structures discussed in the text.

Table 2. Estimated Gain in the Total Absorbed Photon density of the solar cells exploiting the nanostructured $A R C$ with and w/o reflector with respect to a conventional cell (with planar ARC and no reflector), for wavelengths below $(830 \mathrm{~nm})$ and above (900 nm) the GaAs band edge.

\begin{tabular}{|l|l|l|}
\hline \multirow{2}{*}{ Structure } & \multicolumn{2}{|l|}{$\begin{array}{l}\text { Normalized Integrated Absorbed } \\
\text { Photon Density }\end{array}$} \\
\cline { 2 - 3 } & $830 \mathrm{~nm}$ & $900 \mathrm{~nm}$ \\
\hline $\begin{array}{l}\text { Nanocone ARC } \\
\text { w/o reflector }\end{array}$ & 1.2 & 1.3 \\
\hline $\begin{array}{l}\text { Nanocone ARC } \\
\text { with reflector }\end{array}$ & 1.4 & 2.7 \\
\hline
\end{tabular}

The conventional, exponentially decreasing photogeneration in i) is replaced by significant interference patterns in configurations ii) and iii). As highlighted in Table II, both configurations ii) and iii) provide gain in the total absorbed photon density. At $900 \mathrm{~nm}$ (where the GaAs absorption length is $\sim 0.1 \mathrm{~cm}$ ), configuration iii) allows for a factor 2 increase of photogeneration when compared to configuration ii).

The basic structure of the second photonic design, the light-trapping enhanced cell, is shown in Fig. 9. The topmost layer is an ARC which can be realized by a conventional planar thin-film (e.g. silicon nitride $\mathrm{SiNx}$ ) or by the nanostructured "moth eye" layer previously discussed. On the bottom of the cell we have a periodic micro- or nano- structured AlInP layer acting as diffraction grating and a metal mirror ( $\mathrm{Ag}$ in the present simulations) to reflect light as efficiently as possible. By proper optimization of the grating geometrical parameters, light is coupled into high order diffraction modes propagating outside of the escape cone, realizing a strong enhancement of the absorbed photon density. An example of the solar cell absorbance (computed through RCWA simulations) for different thin-film configurations, including both lamellar (2D) triangular gratings and pyramidal (3D) gratings is shown in Fig. 10. The absorbance spectra shows a marked increase around the GaAs band-edge and in the QD range.
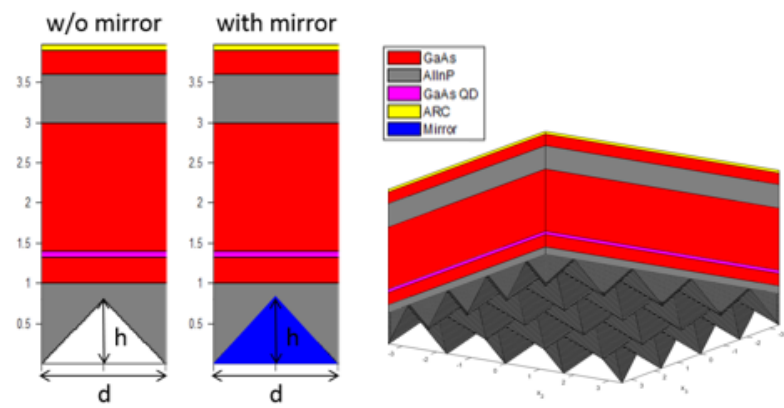

Figure 9. Structure of the thin-film cell with rear-side pyramidal grating.

From the absorbance spectra in Fig. 10 we can estimate the photogenerated current in a specific wavelength range under a specific incident solar irradiance. The grating geometry is optimized to maximize the photocurrent in the QD range (895 nm - $1200 \mathrm{~nm})$. 2D and $3 \mathrm{D}$ diffraction gratings with optimized geometry provide a QD photocurrent of about $1.47 \mathrm{~mA} / \mathrm{cm}^{2}$ and 4 $\mathrm{mA} / \mathrm{cm}^{2}$, respectively, against $0.3 \mathrm{~mA} / \mathrm{cm}^{2}$ and 0.49 $\mathrm{mA} / \mathrm{cm}^{2}$ provided by the single-pass configuration and the thin-film one with planar mirror. Thus, an increase of 5 (2D grating) and 13 (3D grating) times is achieved by the light-trapping enhanced thin-film cells with respect to the conventional wafer-based cell. 


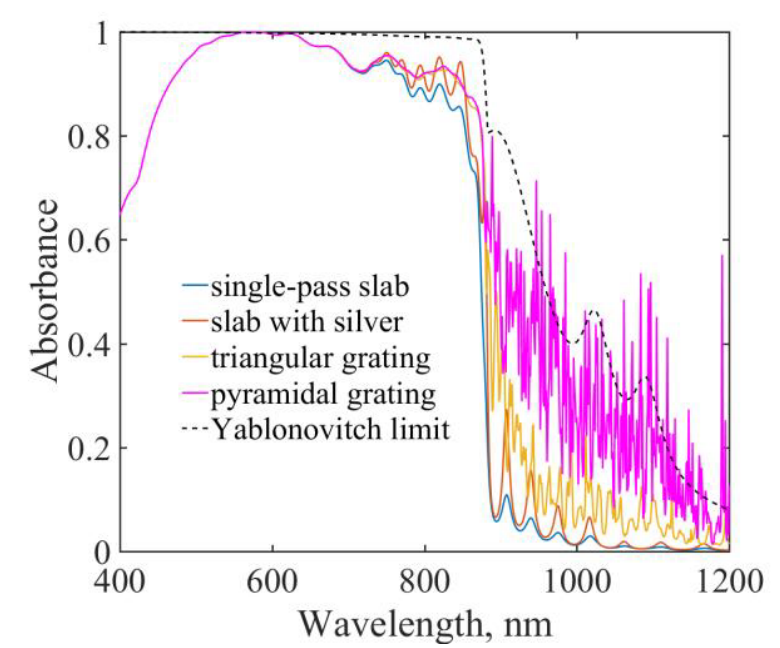

Figure 10. Absorbance spectra of several photonic configurations: wafer-based (single-pass), thin-film with planar silver mirror, thin-film with $2 D$ rear-side triangular grating, and thin-film with rear-side pyramidal grating. The black dashed line indicates the classical Lambertian limit [14].

\subsection{DEVICE-LEVEL SIMULATIONS}

Based on the results in Sec. 3.2, we study here the performance of the QD cell structure described in Sec. 3.1 considering a conventional wafer-based configuration (single-pass), a thin-film configuration exploiting a planar mirror (double-pass) and a thin-film configuration with textured rear grating (i.e. implementing efficient light-trapping). The last one is studied in the so called Lambertian limit [15] (full LT), corresponding in the $1 \mathrm{D}$ electrical simulation presented here to a maximum photogeneration enhancement of about $2 \mathrm{n}^{2}=25, n$ being the GaAs refractive index, in the limit of very weak absorption. Details on the numerical approach may be found in [16]. Residual reflection and shadowing loss are accounted for by a wavelength independent attenuation factor of $5 \%$ of the incident solar irradiance.

The performance of the QD cells are compared to those of a single-junction GaAs cell with the same epitaxial structure, except for the QD stack region which is replaced by a lightly n-doped GaAs region of equal thickness.

The External Quantum Efficiency (EQE) of the regular GaAs cells and QD cells in the various configurations is analysed in Fig. 11. The enhancement of photoconversion in the thin-film configurations is well visible at the GaAs band edge and QD wavelengths. For the sake of model validation, the measured EQE of the first fabricated prototypes - wafer configuration - is shown in Fig. 12, pointing out the good qualitative agreement between simulations and measurements in terms of QD behaviour. On the other hand, in the above gap region, the measured EQE is substantially lower than the predicted one owing to the absence of the ARC and to non-optimal material quality.

Despite the significant increase of short-circuit current achieved by the QD cell with light-trapping thin-film

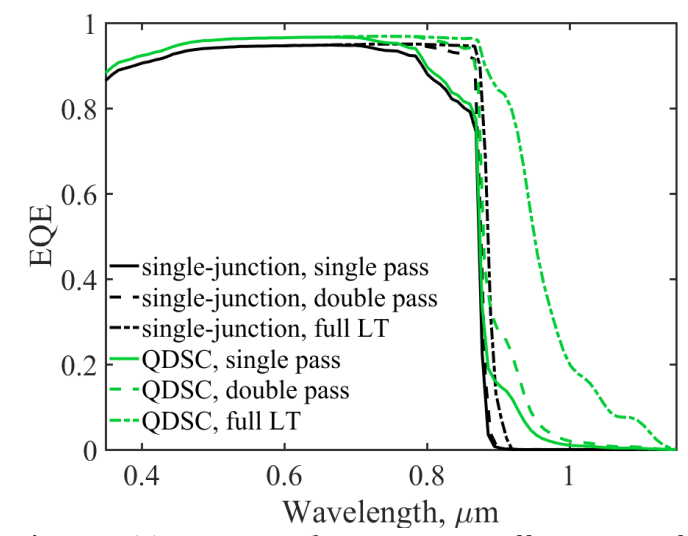

Figure 11. External quantum efficiency of singlejunction and $Q D$ thin film cells, for single-pass, doublepass, and full light-trapping (LT) configurations.

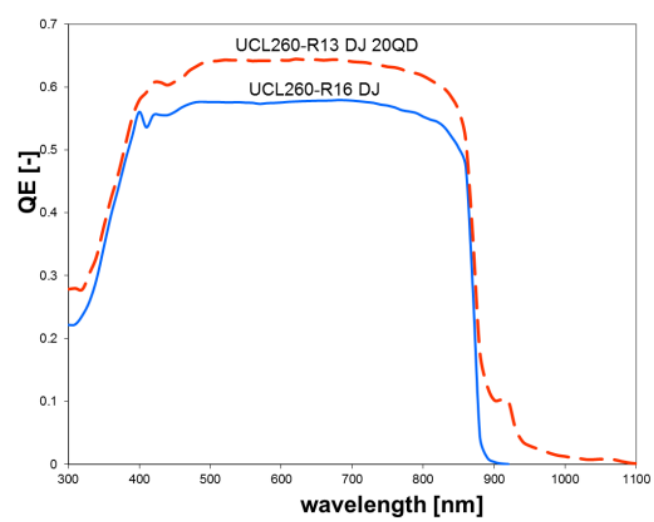

Figure 12. Measured EQE for the first fabricated waferbased prototypes: single-junction (solid line) and 20 QD layers QDSC (dashed line).

configuration, the predicted efficiency (about $24.4 \%$ ) remains substantially lower than the efficiency (26.2\%) of the wafer-based regular cell. The reason must be sought in the significant degradation of the open circuit voltage suffered by the QD cell. This is a very well known - and somewhat controversial - issue of QD cells. One of the main causes for such penalty relies on the inherent nature of QDs as radiative recombination centres under high injection conditions [11]. In fact, at open circuit condition, electrically injected carriers undergo relaxation and radiative recombination through the WL and QD confined states making larger the recombination currents across the cell. One possible strategy to overcome such limitation is by exploiting doping in order to fill the QD states and block relaxation and recombination processes. Indeed, by QD selective doping, substantial mitigation of the open circuit voltage has been experimentally observed in [17], [18], and theoretically explained in view of the suppression of radiative recombination through QDs [12]. An overview of the impact of QD doping (implemented through QD 
direct doping [17]) and of the various photonic configurations on the achievable efficiency of the QD cells is reported in Fig. 13. For the sake of comparison, the efficiency of the corresponding regular GaAs cells is also shown. A significant efficiency increase is achieved in both single-junction and QD thin-film cells with respect to their wafer counterpart. Moreover, in the light-trapping configuration, an absolute improvement of about $0.7 \%$ is achieved by the thin-film QD cell with optimum doping level (around $14 \mathrm{e} / \mathrm{dot}$ ) with respect to the thin-film single junction cell. On the other hand, even though promising, the predicted efficiency values still fall below the target efficiency of $30 \%$. As already mentioned, the recent efficiency record in GaAs solar cells has been achieved by photonic enhancement of the open circuit voltage [5]. In fact, for high quality material, radiative recombination becomes the dominant loss mechanism in the cell and its effective suppression through the so called photon-recycling effect is required to approach the SQ limit. To make significant the reabsorption of the radiative emission and the consequent open circuit voltage enhancement, the emitted photons must be trapped within the cell active region. Owing to the isotropic nature of radiative emission, surface texturing is not necessary, but very high reflectivity at the back-surface mirror is needed to make singlejunction GaAs cells to reach, and possibly overcome, $30 \%$ efficiency [5]. The impact of photon-recycling may be expected to be even more dramatic in high-quality QD solar cells wherein, as previously discussed, the ultimate limit to the attainable photovoltage is set by radiative recombination through the QDs themselves.

Thus, having established a baseline design for the regular and QD cells, we investigate to what extent photon recycling may enhance their maximum achievable efficiency. To this aim, we follow the approach in [10], introducing in the electrical model a radiative recombination rate (both for the bulk and QD recombination rate) that scales inversely with the Photon Recycling (PR) factor, defined as the ratio between the amount of re-absorbed photons and the radiatively emitted photons in the cell active region.
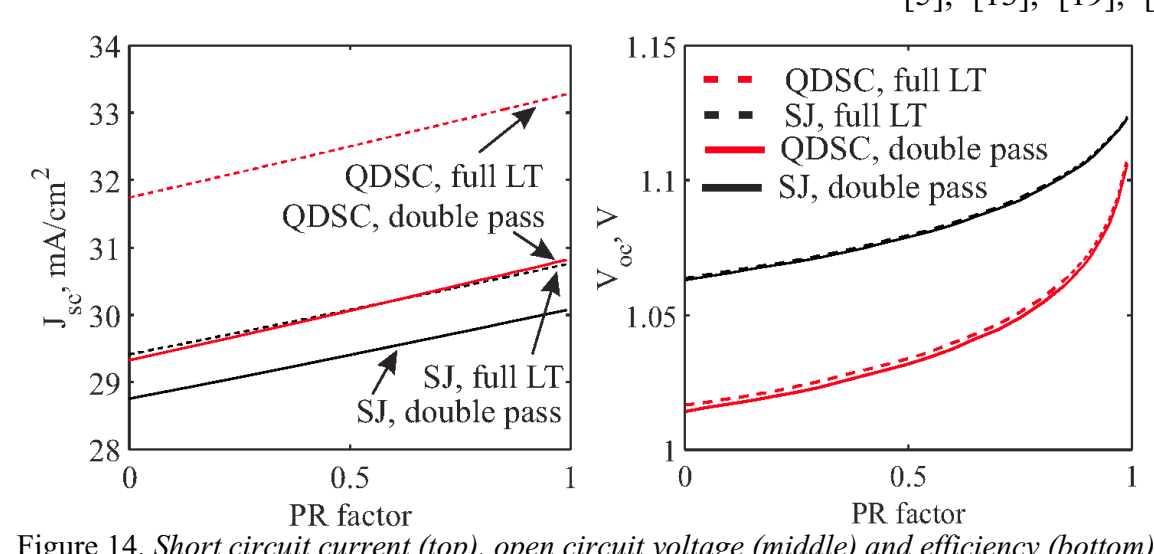

Figure 14. Short circuit current (top), open circuit voltage (middle) and efficiency (bottom) as a function of the photon recycling factor (PR factor) for the single-junction and 20-layers QDSC cells in the double-pass and light-trapping thin-film configurations.

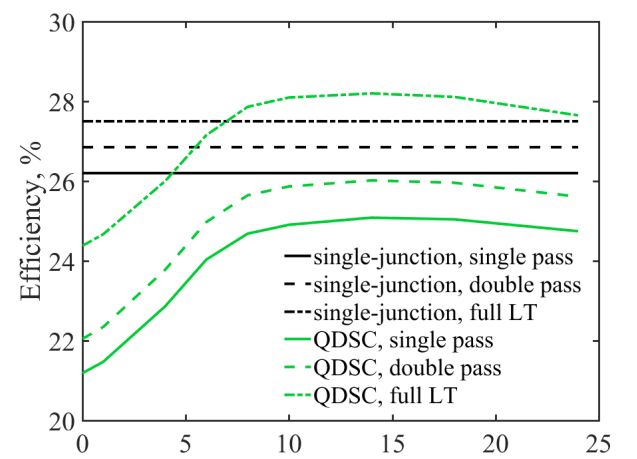

Figure 13. Efficiency of the QD cell vs. QD doping density for the different optical configurations. The horizontal lines show the efficiency of the benchmark single-junction cell.

Clearly, the PR factor depends on the detailed photonic configuration of the cell. Just to focus the ideas, one could notice that some amount of photon recycling is always present even in wafer-based cells and strongly enhanced by placing a high reflective mirror in thin-film devices. Calculations in [10] for a simple $2 \mu \mathrm{m}$ thick GaAs cell report a $80 \%$ PR factor for the wafer-based configuration, and higher than 95\% PR factor for a thinfilm configuration with Au mirror.

Fig. 14 shows the influence of the PR mechanism on the short-circuit current, open circuit voltage, and efficiency of regular GaAs cells and QD cells in the double-pass and light-trapping configuration (including a highly reflective mirror on the backside of the grating, see Fig. 9). As expected, photon-recycling mostly affects the open circuit voltage, with a superlinear increase at high PR factor (markedly stronger in the QD cells with respect to the regular ones). There is also a noticeable effect on the short-circuit current which can be explained in view of slightly improved internal quantum efficiency in the GaAs absorption region. Fill Factor (not shown here) shows instead a negligible increase as the PR factor increases. Similar results for single-GaAs cells with planar optics - i.e. the double-pass configuration - have been already reported by others [5], [13], [19], [20]. Finally, from Fig. 14 we may

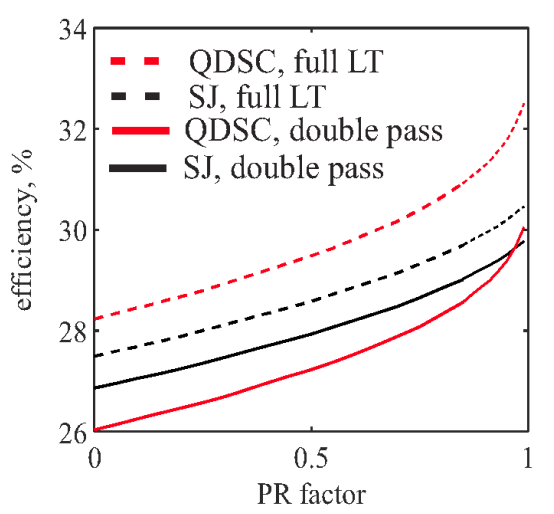

factor (PR factor) for the single-junction and 20-layers QDSC cells in the double-pass and lightrapping thin-film configurations. 
observe that the QD double-pass cell overcomes the single-junction one only for PR factor higher than $97 \%$. On the other end, in the full light-trapping scheme, the QD cell shows higher efficiency than the single-junction one regardless of the PR factor, and overcomes the $30 \%$ target already for PR factor of $70 \%$. Thus, by optimizing material quality, light-trapping scheme and exploiting high reflective mirrors, QD solar cells with efficiency higher than $30 \%$ are feasible.

\section{CONCLUSIONS}

We have presented a novel approach for the realization of high-efficiency, lightweight and flexible space solar cells, based on the integration of promising technologies such as thin-film fabrication via Epitaxial Lift-Off, light-trapping by means of photonic gratings, and nanostructured semiconductors. Efficient photon management within the thin-film device enables to enhance the sun harvesting by quantum dots to such an extent that the quantum dot cell efficiency will be larger than the efficiency of single-junction bulk cells. The engineering of the quantum dot material and of the photonic management structures are discussed. Based on accurate electromagnetic and physics-based simulation simulations, the two processes are considered together in evaluating the performances of the proposed cell through a proof-of-concept design targeting higher than $30 \%$ efficiency.

\section{ACKNOWLEDGEMENTS}

This project has received funding from the European Union's Horizon 2020 research and innovation programme under grant agreement No 687253.

\section{REFERENCES}

[1] M.A. Green, K. Emery, Y. Hishikawa, W. Warta, E.D. Dunlop, "Solar cell efficiency tables (version 47)", Prog. Photovolt: Res. Appl. 24, 3 (2016).

[2] G.J. Bauhuis, P. Mulder, J.J. Schermer: "Thin-film III-V solar cells using epitaxial lift-off” Chapter 21 in: "High-efficiency solar cells: physics, materials and devices" X. Wang, Z.M. Wang (eds.), Springer Int. Pub. (2013).

[3] G.J. Bauhuis, P. Mulder, E.J. Haverkamp, J.J. Schermer, E. Bongers, G. Oomen, W. Köstler, G. Strobl, "Wafer reuse for repeated growth of III-V solar cells", Prog. Photovolt: Res. Appl. 18, 155 (2010).

[4] G.J. Bauhuis, P. Mulder, E.J. Haverkamp, J.C.C.M. Huijben, J.J. Schermer, "26.1\% thin-film GaAs solar cells using epitaxial lift-off", Sol. Energy Mat. Sol. Cells, 93, 1488 (2009).

[5] O.D. Miller, E. Yablonovitch, S.R. Kurtz, "Strong internal and external luminescence as solar cells approach the Shockley-Queisser limit", IEEE J. Photovolt. 2, 303 (2012).

[6] T. Sugaya, O. Numakami, R. Oshima, S. Furue, H.
Komaki, T. Amano, K. Matsubara, Y. Okano, and S. Niki, "Ultra-high stacks of InGaAs/GaAs quantum dots for high efficiency solar cells", Energy Environ. Sci., 5 (3), pp. 6233-6237 (2012).

[7] F. K. Tutu, J. Wu, P. Lam, M. Tang, N. Miyashita, Y. Okada, J. Wilson, R. Allison, and H. Liu, "Antimony mediated growth of high-density InAs quantum dots for photovoltaic cells", Appl. Phys. Lett., 103 (4), p. 043901, (2013).

[8] J. Tommila et al., Sol. Energy Mat. Sol. Cells, 94 (10), pp. 1845-1848 (2010).

[9] T. Aho, A. Aho, A. Tukiainen, V. Polojärvi, M. Raappana, and M. Guina, "Optically enhanced GaInNAs solar cell”, EUPVSEC 2016 (2016).

[10] M.P. Lumb, M.A. Steiner, J.F. Geisz, and R.J. Walters, "Incorporating photon recycling into the analytical drift-diffusion model of high efficiency solar cells," J. Appl. Phys., 116(19), p.194504 (2014).

[11] M. Gioannini et al., IEEE J. Photovoltaics, vol. 3, no. 4, pp. 1271-1278 (2013).

[12] F. Cappelluti, M. Gioannini, A. Khalili. "Impact of doping on InAs/GaAs quantum-dot solar cells: A numerical study on photovoltaic and photoluminescence behavior," Sol. Energy Mat. Sol. Cells, 157, 209-220 (2016).

[13] M. A. Steiner, J. F. Geisz, I. Garcia, D. J. Friedman, A. Duda, 22 and S. R. Kurtz, J. Appl. Phys. 113, 123109 (2013).

[14] E. Yablonovitch and G. D. Cody, "Intensity enhancement in textured optical sheets for solar cells", IEEE Trans. Electron Dev. 29(2), 300 (1982).

[15] Mokkapati, S. and Catchpole, K. R., "Nanophotonic light trapping in solar cells", J. Appl. Phys., 112, 101101 (2012).

[16] F. Cappelluti, et al., "Numerical Study of ThinFilm Quantum-Dot Solar Cells Combining Selective Doping and Light-Trapping Approaches," 43rd IEEE PVSC, Portland, Oregon, (2016).

[17] P. Lam et al., "Voltage recovery in charged InAs/GaAs quantum dot solar cells," Nano Energy, vol. 6, pp. $159-166$, (2014).

[18] S. Polly, D. Forbes, K. Driscoll, S. Hellstrom, and S. Hubbard, "Delta-doping effects on quantum-dot solar cells," IEEE J. Photovoltaics, vol. 4, no. 4, pp. 1079-10 857 (2014).

[19] Wang X, Khan MR, Gray JL, Alam MA, Lundstrom MS. "Design of GaAs solar cells operating close to the Shockley-Queisser Limit," IEEE J. Photovoltaics, Apr;3(2):737-44 (2013).

[20] Walker, A. W., et al. "Impact of Photon Recycling on GaAs Solar Cell Designs," IEEE J. Photovoltaics, 5.6 636-1645 (2015). 